\title{
La mia isola è Las Vegas: laboratorio e testamento letterario
}

Daniel Raffini

\section{(2) OpenEdition}

Journals

Edizione digitale

URL: https://journals.openedition.org/cher/1235

DOI: $10.4000 /$ cher. 1235

ISSN: 2803-5992

Editore

Presses universitaires de Strasbourg

\section{Edizione cartacea}

Data di pubblicazione: 6 novembre 2018

Paginazione: 129-142

ISBN: 979-10-344-0027-0

ISSN: 1968-035X

Notizia bibliografica digitale

Daniel Raffıni, «La mia isola è Las Vegas: laboratorio e testamento letterario», reCHERches [Online], 21 ।

2018, online dal 05 octobre 2021, consultato il 19 novembre 2021. URL: http://

journals.openedition.org/cher/1235; DOI: https://doi.org/10.4000/cher.1235 


\title{
La mia isola è Las Vegas: laboratorio e testamento letterario
}

\author{
Daniel RAFFINI ${ }^{1}$
}

\begin{abstract}
Ta mia isola è Las Vegas, pubblicato postumo nel 2012 a cura di Nicolò LMessina, raccoglie testi brevi di vario genere di Consolo scritti tra il 1957 e il 2011: racconti d'invenzione, rievocazioni storiche, ricordi autobiografici, riflessioni, saggi, episodi di cronaca. Si tratta di testi che in molti casi si pongono sul crinale tra fiction e nonfiction, scritti che partendo da un dato reale - un ricordo autobiografico, una lettura, un evento storico - assumono la veste del racconto vero e proprio, condividendo con esso tecniche e forma. Per spiegare la presenza nel corpus consoliano di testi di questo tipo bisogna tener presente il rapporto complesso che lo scrittore intrattenne con i generi letterari. Nel rifiuto consoliano del romanzo dobbiamo leggere la negazione del romanzo di impostazione tradizionale, il romanzo organico ottocentesco ${ }^{2}$. In un'intervista leggiamo:

Io credo che i miei non siano dei romanzi. Non c'è un'architettura complessa nei miei libri, come nei romanzi ottocenteschi e novecenteschi. È difficile oggi scrivere romanzi. Io ho concepito le mie narrazioni come sorta di narrazioni orali, come poemi narrativi, se vogliamo usare questa cifra aulica, e quindi la mia misura non è quella del racconto breve ma di una narrazione in qualche modo dispiegata. Dico "narrazioni" proprio nel senso benjaminiano, come ho spiegato altre volte, rispetto al romanzo che è tutt'altra cosa. (Traina 2001: 126)
\end{abstract}

1 Daniel Raffini, Università di Roma "La Sapienza".

2 Nel corso di questo intervento si farà riferimento alle opere di Consolo con il termine di romanzi, intendendo questo termine nel senso più vasto che alla parola è stato affidato nel corso del Novecento, quando il romanzo ha finito, in molti casi, per inglobare forme di antiromanzo. La definizione di antiromanzo è stata d'altronde usata da alcuni critici per le stesse opere di Consolo (cfr. Di Legami 2008). Allo stesso modo si userà il termine di racconto per definire i testi spesso ibridi che formano $\mathrm{La}$ mia isola è Las Vegas. 
Il rifiuto del romanzo da parte di Consolo rimanda quindi alla distinzione fatta da Benjamin tra narratore e romanziere ${ }^{3}$. Il passo citato - tralasciando le ulteriori implicazioni benjaminiane - autorizza una lettura di tipo unitario della raccolta La mia isola è Las Vegas. Se la misura dello scrittore non è quella del racconto breve, questi testi vanno allora letti nel loro insieme come una narrazione dispiegata, da integrare alle altre narrazioni di Consolo. Alle ragioni di poetica si uniscono, a favore della lettura unitaria, ragioni pratiche. Infatti, benché i racconti escano in tempi diversi e in diverse sedi e il libro sia postumo, sappiamo che la raccolta fu progettata e voluta dallo stesso Consolo ${ }^{4}$. C'è dunque nella scelta dei testi una volontà autoriale, aumentata dal fatto che nel momento in cui compone la raccolta lo scrittore ha già alle sue spalle l'intera carriera, le riflessioni e le opere maggiori ed è forte di un primo giudizio critico sulla sua opera.

I testi di questa raccolta si offrono come campione esemplare per analizzare la scrittura breve di Vincenzo Consolo. La mia isola è Las Vegas è infatti, insieme a Le Pietre di Pantalica, l'unica raccolta di racconti pubblicata dallo scrittore siciliano - un'esiguità che potremmo senz'altro ricollegare alla predilezione per la narrazione dispiegata di cui si è parlato. Le Pietre di Pantalica mostrano un nucleo unitario forte, costituito dalla sezione Teatro, che deriva da un progetto di romanzo che, iniziato come inchiesta giornalistica sulla vicenda dei frati di Mazzarino, finisce per essere uno spaccato sulla vita del piccolo paese e della Sicilia intera nel secondo dopoguerra ${ }^{5}$. La mia isola è Las Vegas si presenta invece come testimonianza di un'attività letteraria parallela, svolta principalmente su giornali e riviste, ma costante nel corso del tempo e legata a doppio filo alle opere maggiori. Analizzare questa raccolta vuol dire capire in che modo Consolo si sia rapportato alla forma breve, una misura che in molti casi è determinata dalla destinazione in riviste e giornali, ma che assume all'interno del corpus consoliano una sua funzione precisa oltre a un forte valore letterario.

Alla luce di ciò possiamo motivare le due funzioni assegnate alla raccolta nel sottotitolo di questo intervento: laboratorio e testamento, nozioni intorno alle quali ci muoveremo nell'analisi della raccolta consoliana. I racconti di La mia

3 Così lo spiegherà lo stesso Consolo nel saggio I ritorni, pubblicato in Di qua dal faro: «Dico narrazione nel senso in cui l'ha definita Walter Benjamin in Angelus Novus. Dice in sintesi, il critico, che la narrazione è antecedente al romanzo, che essa è affidata più all'oralità che alla scrittura, che è il resoconto di un'esperienza, la relazione di un viaggio. "Chi viaggia, ha molto da raccontare" dice. "E il narratore è sempre colui che viene da lontano. C'è sempre dunque, nella narrazione, una lontananza di spazio e di tempo". E c’è, nella narrazione, un'idea pratica di giustezza e di giustizia, un'esigenza di moralità» (Consolo 2015: 1120).

4 Nella Cronologia del «Meridiano» si legge: «A settembre [del 2004] si avvia il progetto per un volume complessivo dei suoi racconti, la cui cura verrà affidata a Nicolò Messina: uscirà, col titolo La mia isola è Las Vegas, nel maggio 2012, e sarà, benché postumo, un libro a tutti gli effetti ancora di Consolo, che lo segue fino a pochi giorni dalla morte» (Consolo 2015: CXLIV).

5 Cfr. Consolo 2015: 1366-1384. 
isola è Las Vegas possono essere considerati un laboratorio di scrittura perché molti di questi testi brevi sono avantesti e fonti per i successivi romanzi, ma anche spiegazioni e delucidazioni intorno alle modalità di scrittura e alla genesi delle altre opere. Attraverso la forma breve Consolo inizia a lavorare su temi e motivi che poi entrano nelle opere maggiori o torna su ciò che ha già scritto, presentandolo da un nuovo punto di vista. La ricorsività e l'intertestualità interna si pongono dunque come categorie centrali dell'opera letteraria di Consolo: la scrittura ruota attorno a un nucleo ampio ma abbastanza fisso di storie riscritte più volte nel corso degli anni. Allo stesso tempo La mia isola è Las Vegas è anche un testamento letterario, dal momento che ci offre, dalla prospettiva della fine, una visione del mondo consoliano.

In questi racconti è presente sia il Consolo scrittore, che si esprime attraverso tematiche e tecniche, che il Consolo uomo, del quale resta traccia nelle rievocazioni memoriali e autobiografiche per mezzo delle quali è possibile ricostruire un percorso di vita. Pescando dai racconti pubblicati in giornali o rimasti inediti, Consolo ripercorre in questa raccolta i temi principali della sua narrativa: la Sicilia, sia nel suo rapporto personale che come essenza assoluta, la visione della storia, il viaggio, l'impegno sociale, la vita contadina contrapposta al presente, la ricerca linguistica e lo sperimentalismo. La mia isola è Las Vegas ha dunque la capacità di porsi come inizio e come fine dell'opera di Consolo, inizio perché questi racconti furono in molti casi luogo di elaborazione di temi e idee, fine in quanto summa dell'opera dello scrittore e suo ideale testamento.

Per quanto riguarda la circolazione di temi tra i testi di La mia isola è Las Vegas e i romanzi di Consolo, esemplare è il caso del racconto C'era Mussolini e il diavolo si fermò a Cefalù, pubblicato per la prima volta su «Tempo illustrato» il 2 ottobre 1971, in cui si narra un episodio realmente accaduto all'inizio degli anni Venti, ovvero il soggiorno del mago inglese Aleister Crowley a Cefalù con la sua comunità religiosa dedita al satanismo e al libertinaggio: "Fai ciò che tu vuoi sarà il principio fondamentale della legge" era la regola principale del crowleysmo» (Consolo, 2014: 31). L'uso delle fonti, fino alla citazione diretta per la ricostruzione della vita di Crowley, fa di questo racconto, come di tanti altri della raccolta, un esempio di riutilizzo di materiali e scritture di tipo non finzionale all'interno di una struttura i cui strumenti retorici e stilistici rimandano piuttosto a un racconto di tipo finzionale. Questa è d'altronde la maniera prediletta di Consolo di raccontare la storia. Il passaggio dalla ricostruzione storiografica, basata sulle fonti, alla finzione romanzesca avviene nel momento in cui Consolo immagina Crowley a passeggio per le vie di Cefalù, in visita alla cattedrale della città e al Museo Mandralisca'; è qui che l'autore ipotizza l'incontro tra Crowley e il Ritratto d'ignoto di Antonello da Messina, un

6 Il passaggio è segnalato da un cambiamento nell'uso dei verbi, da tempi di carattere assertivo a perifrasi verbali che indicano ipotesi o deduzione: «Nei momenti liberi che gli lasciavano i riti, doveva di certo scendere in paese e visitare la grande cattedrale edificata da Ruggero II il normanno» (Consolo 2014: 32). 
incontro paragonabile a quello tra il ritratto e Mandralisca descritto nel primo capitolo de Il sorriso dell'ignoto marinaio. In entrambi i casi l'osservatore subisce l'effetto straniante del ritratto.

C'era Mussolini e il diavolo si fermò a Cefalù (1971)

E un'altra visita doveva fare l'ex studente di Cambridge, quella al museo Madralisca, dove un piccolo «ritratto d'ignoto» d'Antonello di Messina lo avrà certo irritato più dei mosaici del duomo, e scosso nel suo misticismo e nelle sue credenze. Il sorriso ironico e gli occhi che scrutano profondamente di quel personaggio che esprime tutta la lucida razionalità, l'equilibrio e la pienezza di umanità possibile, saranno certo stati una sfida alla sua irrazionalità, alla sua confusione, ai sentieri oscuri e insondabili delle sue estasi e delle sue allucinazioni. (Consolo 2014: 33)

Il sorriso dell'ignoto marinaio, Capitolo primo (1969; 1976)

L'uomo era in quella giusta età in cui la ragione, uscita salva dal naufragio della giovinezza, s'è fatta lama d'acciaio, che diverrà sempre più lucida e tagliente nell'uso ininterrotto. [...] Tutta l'espressione di quel volto era fissata, per sempre, nell'increspatura sottile, mobile, fuggevole dell'ironia, velo sublime d'aspro pudore con cui gli esseri intelligenti coprono la pietà. [...]

Il personaggio fissava tutti negli occhi, in qualsiasi parte essi si trovavano, con i suoi occhi piccoli e puntuti, sorrideva a ognuno di loro, ironicamente, e ognuno si sentì come a disagio. (Consolo 2015: 144)

La lettura dei due passi evidenzia la persistenza di elementi lessicali e di aree semantiche, che indicano l'insistenza dell'autore sul carattere perturbante del ritratto nei confronti dell'osservatore. In entrambi i casi l'effetto prodotto dalla vista del quadro è un senso di fastidio: Crowley si sente «irritato» e «scosso", mentre Mandralisca e i suoi ospiti si trovano «a disagio». Se la reazione di Crowley è più forte rispetto a quella di Mandralisca, ciò dipende dal fatto che Crowley si caratterizza nel racconto come simbolo dell'irrazionale, mentre l'ignoto del ritratto è emblema di razionalità7. Altro elemento che ricorre nei due testi è il carattere ironico del sorriso dell'ignoto, che contribuisce a creare il senso di disagio nell'osservatore. Ecco dunque un esempio tipico della ricorsività di cui si parlava, del ripetersi di elementi tematici legati fortemente anche a livello lessicale. Difficile stabilire una preminenza cronologica dell'uno o dell'altro

7 L'opposizione tra razionalità e irrazionalità è uno degli elementi tematici forti nell'opera consoliana. Tale dicotomia è vista dallo scrittore come carattere essenziale della Sicilia. Ne troviamo un esempio nel racconto Chesterfield, pubblicato ne Le pietre di Pantalica, in cui il polo della razionalità è occupato da Sciascia e quello della follia da frate Agrippino. Scrive Consolo in questo racconto: «L'uomo [Sciascia], che prima aveva sorriso divertito, si fece serio, triste, scoprendo all'improvviso di trovarsi faccia a faccia col male misterioso e endemico di questa sua terra. E gli capitava spesso, a lui cultore della razionalità, del pensiero chiaro e ordinato, amante dell'ironia e del piacere dell'intelligenza, d'imbattersi in persone che lo incuriosivano per la loro originalità, per la loro comica eccentricità, che gli facevano pregustare spasso, gioco lieve e ameno, e che scoprivano invece, come denudandosi all'improvviso, la malata pelle della pazzia. E gli si rivoltava allora tutto in amaro, in penoso» (Consolo 2015: 566). 
testo, ma in ogni caso ci troviamo di fronte a un esempio evidente e tipico di circolazione di immagini tra i testi brevi e i romanzi di Consolo ${ }^{8}$.

Sicuramente posteriore è invece la ripresa del personaggio di Crowley nel romanzo Nottetempo, casa per casa del 1991. Il racconto qui si pone in un momento cronologico molto anteriore e conferma la presenza di Crowley nell'immaginario consoliano già vent'anni prima della pubblicazione del romanzo. Sappiamo che Nottetempo, casa per casa fu scritto da Consolo in breve tempo, a ridosso della pubblicazione, ma anche che appunti e studi su vari temi presenti nel libro appaiono nell'Archivio Consolo fin dalla fine degli anni Sessanta, in coincidenza quindi con la stesura di C'era Mussolini e il diavolo si fermò a Cefalù ${ }^{9}$. Ci limiteremo, in questa sede, ad osservare che nel romanzo torneranno molti degli episodi accennati nel racconto, tra cui la visita di Crowley alla Cattedrale di Cefalù.

Questo racconto dimostra come i testi di Consolo risultino spesso strettamente legati tra loro. Alla base di tale correlazione ci sono le ricerche storiche dello scrittore e l'interesse per episodi e vicende minori della storia. La frequentazione con le storie e le microstorie siciliane inizia già negli anni Cinquanta e a questa passione è legato l'inizio dell'amicizia con il poeta Lucio Piccolo, come lo stesso Consolo racconta in Piccolo grande Gattopardo:

Nel negozio degli Zuccarello dunque - era il 1954 - incontrai Piccolo. Il quale, dopo aver consegnato le sue poesie, appuntò gli occhi sui miei libri che avevo portato là per farli rilegare - vecchi libri che scovavo sulle bancarelle: storie locali, guide, almanacchi... - «M'accorgo di non essere il solo ad amare questi libri» disse, "gli almanacchi, le guide, sono pieni di insospettabile poesia». E aggiunse: «Ho un'intera biblioteca di questi libri. Venga, venga a trovarmi». (Consolo 2014: 210)

Da questa passione deriva il tesoro di storie dimenticate che formano l'intelaiatura di molte opere di Consolo. Se la scrittura di Consolo si basa in buona parte su questo repertorio, dall'altro lato c'è il contributo altrettanto importante dell'esperienza personale. In questo senso è possibile ad esempio ricostruire la genesi, tutta personale e autobiografica, del capitolo In Mozia de' Fenici di Retablo, in cui viene raccontata la visita di Clerici e Isidoro a Mozia e il trafugamento della statua greca con conseguente naufragio. Episodio apparentemente romanzesco, ma dietro al quale si cela l'esperienza personale dello scrittore. Nel racconto Le vele apparivano a Mozia, pubblicato per la prima volta nel 1988 e poi confluito in La mia isola è Las Vegas, Consolo racconta la

8 Il racconto è pubblicato per la prima volta nel 1971, mentre nel 1969 era stato pubblicato il primo capitolo de Il sorriso dell'ignoto marinaio sulla rivista "Nuovi Argomenti»; tuttavia in Consolo non sempre i tempi di composizione coincidono con quelli di pubblicazione. Per notizie sulla genesi de Il sorriso dell'ignoto marinaio cfr. Consolo, 2015: 1299-1321 e gli studi di N. Messina e D. O'Connell. Per maggiori precisazioni sui tempi di stesura di C'era Mussolini e il diavolo si fermò a Cefalù, sarebbe invece necessario un confronto con manoscritti e dattiloscritti eventualmente conservati nell'Archivio Consolo.

9 Cfr. Consolo 2015: 1389; Messina 2007: 60-65. 
sua esperienza personale sull'isola fenicia, da cui derivano molti elementi di quella immaginaria di Clerici e Isidoro. Siamo nel 1984, Consolo ha partecipato a un matrimonio a Palermo con Guttuso e altri pittori. Viene ricordato l'autistainserviente-guardiano di Guttuso, che curiosamente si chiama proprio Isidoro. Dopo il matrimonio Consolo e altri partono per un itinerario archeologico in Sicilia. Il viaggio, a completare le corrispondenze onomastiche, avviene, tra gli altri, proprio con quel Fabrizio Clerici che darà il nome al protagonista di Retablo. Il primo incontro dei viaggiatori sull'isola è quello con la statua greca dell'efebo, la stessa che Isidoro e Clerici trafugano in Retablo. Si nota dunque una particolare consonanza tra l'esperienza personale e ciò che diventerà scrittura finzionale in Retablo. Sempre ne Le vele apparivano a Mozia, Consolo, vedendo la statua, torna con la mente alla sua prima visita nella piccola isola fenicia, quando, appena finito il liceo, aveva intrapreso un viaggio in solitaria alla scoperta della Sicilia. Anche da questo più antico viaggio Consolo prende spunto per Retablo. Ne è un esempio la narrazione dell'incontro del giovane Consolo con il custode dell'isola:

Il Caronte, un contadino-custode, si divertì molto nel tragitto a raccontarmi che l'isola era infestata da "spiriti", che sotto le antiche pietre stavano intanati fantastici serpenti, fra gli alberi e gli arbusti erano annidati civette, gufi millenari, che dal basso mare intorno alzavano la cresta orrende dragonesse. (Consolo 2014: 126)

Così in Retablo Isidoro, arrivato a Mozia, vedendo le anfore sotterrate e le steli della necropoli, esclama: «Ma dove capitammo? [..] a voler dire il vero, questa mi sembra un'isola di spirti: questo campo, don Fabrizio, sarìa incantato, sarìa questo seminato di pignatte una grande trovatura? O stiamo noi sognando?» (Consolo 2015: 444). Di particolare rilevanza è poi l'immagine delle vele dei Fenici che, grazie a un salto temporale, appaiono davanti agli occhi del giovane Consolo così come di fronte allo sguardo di Clerici ed Isidoro: «Fu quel giorno, in quell'ora, alla Porta Sud, in piedi sull'argine del Cothon, l'intatto, rettangolare bacino artificiale costruito dai Fenici, guardando il mare, ch'io vidi all'orizzonte le snelle barche dalle vele quadrate» (Consolo 2014: 127). In un altro racconto, La grande vacanza orientale occidentale, Consolo ritorna su quel primo viaggio a Mozia e scrive ancora: «Al sole poi, davanti a quel mare stagnante, mi sembrava di veder sopraggiungere, a frotte, le snelle barche dalle vele porpuree, il grande occhio apotropaico dipinto sulle prore» (Consolo 2014: 167). E l'immagine è anche in Retablo: «Ecco che da riviere s'aprono le distese dei mari che naviganti improvvidi solcarono e notti illuni e cieli gravi» (Consolo 2015: 442).

Mozia isola Fenicia. In quest'ottica quella statua greca, la cui presenza nemmeno gli studiosi riescono a spiegare, diventa un'intrusa. Consolo lo percepisce subito, fin da quel lontano viaggio da ragazzo, e quella antica impressione lo spinge in Retablo a far trafugare la statua da Clerici:

Per questa mia memoria della prima visita nell'intatta Mozia, in un mio racconto, Retablo, volli portar via dall'isola la statua greca [...] Portarla via e farla naufragare, sparire in fondo al mare: come contrappasso o compenso alla morte per acqua del giovane fenicio Phlebas [...] di eliotiana creazione; perché quella statua di 
marmo mi sembrò una discrepanza, un'assurdità, una macchia bianca nel tessuto rosso della fenicia Mozia; mi sembrò una levigatezza in contrasto alla rugosità delle arenarie dei Fenici; uno squarcio, una pericolosa falla estetica nel concreto, prammatico fasciame dei mercanti venuti dal Levante. (Consolo 2014: 127)

Di questo discorso, che sta dietro la scelta narrativa, rimane in Retablo solo la breve riflessione di Clerici, che, notando la matrice classica della statua, scrive che essa è «dunque stranea qua, questa scoltura, inusitata, in quest'isola di Mozia, in mezzo a questi resti punici e fenici» (Consolo 2015: 451). In questo caso, dunque, attraverso la scrittura breve dei racconti lo scrittore motiva e giustifica le scelte narrative dei romanzi.

Gli esempi illustrati - a cui se ne potrebbero aggiungere altri - dimostrano come molti testi di La mia isola è Las Vegas possano aprire le porte del laboratorio dell'autore, permettendo di capire le scelte intraprese nel corso della scrittura. Consolo, in effetti, parla molto di se stesso e delle proprie opere: egli si racconta e ci racconta il suo lavoro, e il luogo privilegiato di questa autonarrazione sembrano essere proprio i testi brevi apparsi nel corso degli anni in giornali e riviste. Bisogna poi almeno accennare al fatto che all'intertestualità interna si aggiunge l'intertestualità esterna, le fonti di vario tipo - letterarie, storiche, saggistiche - utilizzate da Consolo per la costruzione dei suoi testi, un rapporto che arriva in alcuni casi fino alla citazione diretta, e che fa di lui uno scrittore «palincestuoso» ${ }^{10}$.

In alcuni dei testi de La mia isola è Las Vegas Consolo riflette di poetica, del suo modo di intendere la letteratura. Tra la varie riflessioni spiccano quelle sulla scrittura e quelle sulla storia; esemplare in questo senso è il racconto-saggio Un giorno come gli altri. Il primo punto su cui porre l'attenzione è la presenza di un discorso sotterraneo sui generi letterari e sulle modalità di scrittura:

Riprendo a lavorare a un articolo per un rotocalco sul poeta Lucio Piccolo. Mi accorgo che l'articolo mi è diventato racconto, che più che parlare di Piccolo, dei suoi Canti barocchi, in termini razionali, critici, parlo di me della mia adolescenza in Sicilia, di mio nonno, del mio paese: mi sono lasciato prendere la mano dall'onda piacevole del ricordo, della memoria. (Consolo 2014: 92)

Qui Consolo conferma la labilità dei confini dei generi e presenta la sua come una scrittura fluida, che scivola da una modalità all'altra senza soluzione di continuità. Il saggio a cui fa riferimento nel passo citato è probabilmente Il barone magico, pubblicato ne Le pietre di Pantalica, che in effetti più che un saggio su Piccolo è la rievocazione dei momenti condivisi. D'altronde lo stesso Un giorno come gli altri ci mostra il processo di trapasso apparentemente inconsapevole da un genere all'altro sul filo della memoria e della riflessione. Il testo inizia come racconto realistico, in parte autobiografico: narra della storia di Turi, un ragazzo siciliano emigrato a Milano, che vive in gravi ristrettezze economiche, e descrive il clima degli anni di piombo e le perquisizioni della polizia. Il racconto però si interrompe all'improvviso, sostituito da una riflessione sul rapporto tra arte e

10 Cfr. O’Connell 2008; Cuevas 2000. 
politica, per scivolare infine verso speculazioni letterarie. In quest'ultima parte si inserisce il passo citato in cui Consolo descrive il farsi del saggio su Lucio Piccolo. Tale processo di passaggio dal racconto al saggio, o più in generale da un genere a un altro, è ravvisabile in molti testi dello scrittore ${ }^{11}$. Un giorno come gli altri si presenta dunque come un racconto doppiamente metaletterario: per il suo riflettere sulla scrittura e per essere esso stesso un esempio del procedimento su cui riflette. In questa sede, inoltre, Consolo introduce la distinzione tra narrare e scrivere ${ }^{12}$, da cui emerge l'importanza della scrittura nel suo potere di intervenire sul mondo, un punto della poetica consoliana che emerge nei testi de La mia isola è Las Vegas e che leggiamo espresso chiaramente in Memorie:

La prosa dunque della narrazione nasce per me da un contesto storico e allo stesso contesto si rivolge. Si rivolge con quella parte logica, di comunicazione che sempre ha in sé il racconto. Che è, per questa sua origine per questo suo destino, un genere letterario "sociale". Sociale voglio dire soprattutto perché, in opposizione tematica e linguistica al potere, responsabile del malessere sociale [...] il narratore vuole rimediare almeno l'infelicità contingente. (Consolo 2014: 92)

Arriviamo così all'altro punto teorico importante affrontato in questi testi: il rapporto con la storia. Nel racconto La testa fra i ferri della ringhiera del 2003 Consolo parla del punto di vista della storia, un punto di vista secondario e defilato. La testa fra i ferri della ringhiera racconta la storia d'Italia, da Mussolini fino ad oggi, vista dal balcone di piazza Vittorio Emanuele a Sant'Agata di

11 Un altro caso interessante di scivolamento da un genere all'altro è la sezione Teatro de Le pietre di Pantalica, derivata da un iniziale lavoro di tipo giornalistico. Scrive Consolo a tale proposito: «L'idea prima del libro era quella di scrivere un romanzo su un fatto di cronaca che riguardava i frati del convento di Mazzarino, processati per mafia: mandavano lettere minatorie e minacciavano i ricchi del paese per avere in cambio denaro. Mi recai quindi a Mazzarino, paese al centro della Sicilia [...], per conoscere i luoghi e documentarmi sulla vicenda criminosa. Ma là scoprii che Mazzarino era stato il luogo emblematico delle lotte contadine del Secondo dopoguerra, delle occupazioni delle terre dei feudatari, della repressione da parte dello Stato, della sconfitta e quindi del fallimento della riforma agraria, con la beffa finale (Filosofiana) e la condanna dei contadini all'emigrazione nel nord Italia e nel Centro d'Europa. Questa epopea contadina mi interessò di più della vicenda dei frati mafiosi. E così, sotto forma di racconti, scanditi in tre parti (Teatro - Persone Eventi) ho voluto raccontare pasolinianamente la fine del mondo contadino in Sicilia. [...] raccontare dell'arrivo degli americani (Lo Sherman, Il fotografo - Robert Capa -) le rivolte popolari, la renitenza alla leva dei richiamati alle armi dopo l'8 settembre (La volpe di donna Elisa)» (Consolo 2015: 1369).

12 «E che il narrare, operazione che attinge quasi sempre alla memoria, a quella lenta sedimentazione su cui germina la memoria, è sempre un'operazione vecchia arretrata regressiva. Diverso è lo scrivere, lo scrivere, per esempio, questa cronaca di una giornata della mia vita il 15 di maggio del 1979: mera operazione di scrittura, impoetica, estranea alla memoria, che è madre della poesia, come si dice. E allora è questo il dilemma, se bisogna scrivere o narrare. Con lo scrivere si può forse cambiare il mondo, con il narrare non si può, perché il narrare è rappresentare il mondo, cioè ricrearne un altro sulla carta» (Consolo 2014: 92). 
Militello ${ }^{13}$, dove lo scrittore ha vissuto la sua infanzia e la sua adolescenza. Da quel balcone Consolo assiste alle adunate fasciste, all'annuncio dell'entrata in guerra, all'occupazione tedesca, all'arrivo degli americani, al ritorno del fratello dalla guerra, ai comizi per le elezioni regionali, di lì vede i socialisti e i democristiani, scioperi e cortei, e conclude:

Vidi e vidi, da quel mio balcone, in quel lontano tempo della mia infanzia e della mia adolescenza. E dico oggi che la grande storia, la Storia, passa anche, spesso, dai luoghi più ignoti, può mostrare la sua faccia anche in un piccolo spazio, quale può essere la piazzetta di uno sperduto paesino, può essere osservata dagli occhi ancora limpidi di un fanciullo ed imprimersi indelebilmente nella sua memoria. E può essere, la storia, da quel fanciullo divenuto adulto, anche narrata. Non nel modo scientifico degli storici di professione, ma in tutt'altro modo. (Consolo 2014: 204)

Consolo teorizza così un nuovo punto di vista sulla storia, che si concretizza nei suoi romanzi nella rivalutazione di episodi e personaggi minori e un nuovo modo di raccontare gli eventi, diverso da quello della storiografia ufficiale. Nei racconti di Consolo spesso emerge tra le righe una critica alla storiografia, alla scienza della storia e alle sue versioni ufficiali ${ }^{14}$. Nel racconto Le perizie del 1976 Consolo racconta una vicenda rocambolesca: un incisore ruba in un ossario un teschio risalente al 1780 per disegnarlo, poi lo getta in un cortile, che si rivela essere quello di un mafioso, che a sua volta lo getta in mare, dove viene ripescato da un pescatore. I carabinieri chiamano i periti, che datano il teschio a sei o sette anni prima. Conclude allora Consolo:

Le perizie. Sempre diffidarne, anche di quelle di eminenti specialisti, di insigni studiosi, di luminari, di massime autorità del campo. Lo stravolgimento della realtà, l'errore storico si annida sotto. I reperti sono sempre più vecchi, più di quanto risulti dalle minuziose analisi.

L'attuale crisi economica italiana, questa testa o teschio di oggi, per esempio, a quando risale, appartiene a un antico cadavere mummificato? (Consolo 2014: 55)

La storia va sempre messa in discussione e il presente con essa. L'invito è a scavare nelle cause, in ciò che c'è dietro un evento, senza fermarsi a un giudizio che potrebbe essere un pregiudizio. Solo così la storia può essere utile, nel suo senso originario di indagine. In un mondo in cui il bene e il male si mescolano fino a non riconoscersi più, la visione manichea offerta dalla storiografia ufficiale non può più essere accettata; è quello che dice Consolo in un passo de La grande vacanza orientale-occidentale nel 1999 in cui commenta il giudizio favorevole di Montesquieu sul fatto che i siracusani abbiano interrotto l'usanza fenicia di sacrificare fanciulli:

Ammirevole sì, quel trattato, ma l'illuminato barone francese dimenticava che quegli stessi Siracusani, dopo la vittoria, avevano crocifisso tutti i greci che avevano combattuto accanto ai Fenici-Cartaginesi. È crudeltà, massacro, orrore dunque la storia? O è sempre un assurdo contrasto? Quei Fenici che sacrificavano

13 La piazza è oggi intitolata allo scrittore.

14 Cfr. Segre 2005. 
i loro figli agli dèi erano quelli che avevano inventato il vetro e la porpora, e la scrittura segnica dei suoni, aleph, beth, daleth... l'alfabeto che poi usarono i Greci e i Latini, usiamo anche noi, quei Fenici che, con i loro commerci, per le vie del mare portarono in questo Mediterraneo occidentale nuove scoperte e nuove conoscenze. (Consolo 2014: 67) ${ }^{15}$

In questa visione viene meno anche la tradizionale concezione di eroismo, vengono rimessi in discussione i termini del discorso e riaffermata la difficoltà di sciogliere il nodo bene-male.

Oltre ad essere un momento importante di riflessione teorica, la storia si concretizza in questi racconti come contenuto vero e proprio. Attraverso questi testi ripercorriamo le vicende della Sicilia e dell'Italia dall'Unità fino al berlusconismo. Consolo si concentra su episodi e personaggi "minori" della storia, come le rivolte siciliane del 1860 (E poi arrivó Bixio, angelo della morte), la storia politica della Sicilia del dopoguerra (Alèsia al tempo di Li Causi), le lotte sindacali (Un filo d'erba al margine del feudo) o le affascinanti imprese degli stranieri in Sicilia (Orazio Nelson, Aleister Crowley, Joseph Withaker).

Il discorso sulla storia non è in Consolo un discorso di tipo archeologico. La riflessione sul passato è una riflessione sul presente, unico punto di arrivo di ogni speculazione dello scrittore. Nel confronto con il passato, il presente rivela infatti un inarrestabile cammino di dissoluzione, il progressivo avvicinamento di ogni civiltà alla propria fine. Nel racconto Il disastro storico del 1978 Consolo parla dello sciacallaggio, che spesso segue la distruzione portata da una calamità naturale. Parlando di questi eventi l'autore scrive che, oltre a produrre molte vittime, essi sono anche un "disastro della storia», in quanto distruggono "città o tessuti umani fortemente storicizzati, che nei secoli avevano cioè sviluppato una loro particolare storia, una loro cultura, una loro civiltà, oltre a distruggere vite e documenti e beni, ributta indietro i superstiti dal piano della storia al piano della natura, dell'esistenza: in pochi secondi essi fanno balzi indietro di secoli» (Consolo 2014: 67).

La storia di un luogo è allora la sua cultura, l'azione umana che agisce su uno spazio. Per questo il tramonto di una cultura può determinare la fine della storia. In alcuni dei racconti riuniti in La mia isola è Las Vegas Consolo focalizza l'attenzione su questo momento di passaggio. In Porta Venezia scrive: «Io trapiantato qui, come tanti, da un sud dove la storia si è conclusa, o come questi africani, da una terra d'esistenza (o negazione d'esistenza) dove la storia è appena o non è ancora cominciata» (Consolo 2014: 113). Nel 1988, quando scrive questo racconto, Consolo registra dunque per la Sicilia la fine della storia, il tramonto del mondo contadino, quel mondo che nei racconti degli anni Cinquanta e Sessanta Consolo descriveva come vivo. Nei primi testi di La mia isola è Las Vegas si registra infatti un ritorno insistente sulle credenze e sulle tradizioni popolari. In Un sacco di magnolie, del 1957, un ragazzo viene sottoposto al rito per scovare il malocchio, mentre nel racconto successivo, un inedito riportante

15 Il passo è ripreso anche in Retablo (Consolo 2015: 446). 
la data 1960-1970, l'autore rievoca la tradizione dei dolci dei morti. Il tema funebre, motivo fortemente popolare, appare spesso in questi primi racconti: in Grandine come neve viene narrato il doppio funerale di un padre e di un figlio, mentre in Un filo d'erba al margine del feudo il narratore racconta la visita alla camera ardente del socialista Carmelo Battaglia, assassinato nel 1966. Più tardi, a partire dagli anni Ottanta, i testi di Consolo iniziano a registrare il tramonto della civiltà contadina, la sua trasformazione, determinata in gran parte dalla ferita dell'emigrazione, tema che diventa dominante. Emigrazione di cui è protagonista lo stesso Consolo, ma di cui - dice in Porta Venezia - siamo in fin dei conti protagonisti tutti ${ }^{16}$. La Milano raccontata dallo scrittore è una città multietnica, una società in cui le provenienze si mescolano, in un catalogo di usanze e tradizioni, modi di vivere differenti che si trovano a convivere in uno spazio ristretto.

La Sicilia è invece verghianamente vittima del progresso, della modernità che investe e pone fine a un mondo millenario, che affonda le sue radici nel periodo arabo e in quello normanno. Nel racconto Arancio, sogno e nostalgia Consolo ripercorre la storia della Sicilia attraverso la storia di uno dei suoi simboli ${ }^{17}$. L'arancio assume valore mitico, dal momento che gli agrumeti sarebbero stati impiantati sull'isola in epoca musulmana. La tradizione della coltivazione degli agrumi, con le sue tecniche, conosciute da poche famiglie di vivaisti siciliani, diventano in questo racconto simbolo del mondo contadino e tradizionale che va scomparendo sotto gli occhi di chi narra: «Si parla di tempi andati, di tempi d'oro, tempi che sono durati fino all'altro ieri» (Consolo 2014:133). L'arancio è schiacciato dall'irruzione della modernità, del mercato mondiale e delle tecniche di produzione di massa e diventa allora l'emblema di un mondo che sta scomparendo. In due racconti più recenti, del 2007 e del 2008, il discorso arriva a piena maturazione attraverso l'appropriazione del concetto pasoliniano di mutamento antropologico. Parlando degli anni in cui studiava a Milano, Consolo scrive:

Erano quelli gli anni della fine del mondo contadino e della rapida trasformazione dell'Italia in Paese neo-industriale, del miracolo economico e della mutazione antropologica; gli anni, quelli dell'espulsione dal Paese di milioni e milioni di lavoratori in cerca d'un futuro, d'un destino migliore. (Consolo, 2014: 226)

Un tema particolarmente caro a Consolo, che troviamo anche ne Le pietre di Pantalica. Qui Antonino Uccello, poeta-etnologo amico di Consolo, si oppone al declino del mondo contadino cercando di salvarne le vestigia, raccogliendo gli strumenti e le testimonianze; oggetti che trova abbandonati nelle campagne,

16 Durante una retata all'interno di un locale di egiziani, il narratore vorrebbe urlare al poliziotto che gli chiede se è italiano: «No, no, sono negro, sono arabo, sono ebreo, sono di tutte le razze, come te!» (Consolo 2014: 116)

17 Quello di prendere un prodotto o un'attività come simbolo del mondo siciliano è un procedimento ravvisabile anche nei racconti-reportage Uomini e paesi dello zolfo e $\mathrm{La}$ pesca del tonno pubblicati in Di qua dal faro. 
relitti della storia, il cui unico destino rimasto è quello di essere musealizzati. Emblema di un mondo che non esiste più è anche il poeta Lucio Piccolo, tanto che nel momento della sua scomparsa il dolore che Consolo prova non è solo per la perdita dell'amico e maestro, ma anche "per un mondo, un passato, una cultura, una civiltà che con lui se ne andavano» (Consolo 2014: 214).

I frequenti riferimenti alla propria vita, dimostrano come la storia di tutti si affianchi nei racconti di La mia isola è Las Vegas alla storia personale di Consolo. Storia e autobiografia si mescolano attraverso l'identità dello sguardo, quella testa che dalle sbarre della ringhiera osserva il mondo. Attraverso questi racconti è possibile ricostruire la vita dello scrittore: l'infanzia in paese (Befana di novembre, Natali sepolti, La testa tra i ferri della ringhiera), i viaggi in camion per la Sicilia con il padre (Le lenticchie di Villalba, Le macerie di Palermo), la prima esplorazione dell'isola in solitaria (Le vele di Mozia, La grande vacanza orientaleoccidentale), gli studi a Milano (Grandi carriere di vecchi amici, Genova, Fenicia d'Occidente), quella Milano che in seguito diventerà la sua residenza (Porta Venezia), l'esperienza alla Rai (Il corista a domicilio, La pallottola in testa), la carriera di scrittore (Porcacchia facean di nome, Memorie, Premio alla carriera), le amicizie con gli altri scrittori (Piccolo grande Gattopardo), i frequenti ritorni in Sicilia (Ritorno al paese perduto, La grande vacanza orientale-occidentale) e gli altri viaggi in giro per il mondo (Madre Coraggio). Al di là del racconto autobiografico, c'è sempre la volontà di parlare della storia, quella di tutti, della Sicilia e d'Italia e la riflessione sui temi cari allo scrittore.

Nel quadro di questo vasto resoconto di vita, ci concentreremo su alcuni racconti degli anni Duemila, in cui si nota una forte tendenza alla rievocazione del mondo dell'infanzia, tra il Fascismo e la guerra. Nei racconti degli ultimi anni l'elemento autobiografico diventa tema quasi unico. Lo scrittore decide di raccontare la sua infanzia, ne fissa su carta i ricordi, in un'operazione di salvataggio di un passato personale minacciato, destinato, come tutte le storie personali, all'oblio. Particolarmente interessanti sono due racconti che ruotano attorno alla figura del padre ${ }^{18}$. Ne Le lenticchie di Villalba Consolo racconta di uno dei primi viaggi in camion, verso il paese di Villalba. Qui il padre si trova a mercanteggiare per l'acquisto di un lotto di lenticchie, ma il maresciallo blocca la partenza: a causa della carestia il carico non può lasciare il paese. Il venditore allora si rivolge al capomafia Calò Vizzini e ottiene la partenza della merce, ma il padre si oppone, rifiuta l'intercessione della mafia e dice al figlio: "Lo vedi? In questi paesi ci sono persone che comandano più dei marescialli. Quando tornerai a scuola, lo scriverai questo in un bel copiato». Un invito che sarà raccolto da Consolo a più di mezzo secolo di distanza, con la scrittura del

18 Pochi sono i riferimenti al padre nell'opera di Consolo. Ad eccezione di questi ultimi racconti, la figura paterna appare solo nella dedica del primo romanzo, La ferita dell'aprile, e attraverso alcuni riferimenti nel racconto I linguaggi del bosco, pubblicato ne Le Pietre di Pantalica. 
racconto: «Il copiato, più o meno bello, è questo che ho scritto qui, dopo quasi sessant'anni» (Consolo 2014: 174).

Nell'altro racconto, Le macerie di Palermo, il piccolo Vincenzo visita per la prima volta il capoluogo siciliano devastato dalla guerra, dove il padre deve consegnare delle pesche per conto di un commerciante di Sant'Agata, Don Saro, il quale chiede anche di cercare notizie di alcuni suoi familiari a seguito del conflitto. Trovatili, il padre apprende della condizione di indigenza in cui versano e decide di lasciargli l'incasso della vendita delle pesche di Don Saro. Ancora una volta il padre invita il figlio a raccontare questa storia: «Poco prima d'arrivare al paese, mio padre si girò verso di me. "A scuola" disse, farai un bel copiato di questo viaggio» (Consolo 2014: 189).

Da questi racconti emerge in modo chiaro la figura del padre con i suoi insegnamenti, alla cui memoria Consolo, in vecchiaia, decide di dedicare la sua scrittura, raccogliendo un antico invito fino ad allora trascurato. Come tanti altri protagonisti delle rievocazioni di Consolo, anche il padre diventa rappresentante di un mondo ormai scomparso e si staglia con la sua moralità spontanea e umile contro l'amoralità della storia. Ancora una volta inizio e fine si sovrappongono e, nell'affacciarsi alla vecchiaia - complice anche il nuovo impegno sociale che lo vede protagonista in questi anni - Consolo torna a guardare alla propria infanzia, a quel mondo perduto e ai valori rappresentati da esso. Ciò che rimane alla fine di tutto è allora il valore etico della scrittura, in rispota alla sempre maggiore spettacolarizzazione dell'informazione e della cultura. Raccontare la storia, ricordare la propria esperienza, rievocare le figure degli amici, raccontare un mondo che non c'è più: tutto questo rientra per Consolo nel dovere morale dello scrittore, e i racconti riuniti come ultimo gesto in La mia isola è Las Vegas ne sono una bellissima testimonianza.

\section{Bibliografia}

Adamo G., 2014, «La storia nell'opera di Vincenzo Consolo», Esperienze letterarie, XXIX, p. 3-18.

Consolo V., 2014, La mia isola è Las Vegas, a cura di Nicolò Messina, Milano, Mondadori.

Consolo V., 2015, L'opera completa, a cura e con un saggio introduttivo di Gianni Turchetta e uno scritto di Cesare Segre, Milano, Mondadori, «I Meridiani».

Cremante R., 2007, «La sperimentazione di Vincenzo Consolo fra storia e invenzione», in: Vincenzo Consolo: punto de unión entre Sicilia y España. Los treinta años de «Il sorriso dell'ignoto marinaio», a cura di Irene Romera Pintor, Valencia, Generalitat valenciana y Universitat de Valencia.

Cuevas M.A., 2000, «La constante metaficcional en la obra de Vincenzo Consolo», in: Una veridade ascosa sotto bella menzogna... Zur italienischen Erzählliteratur der Gegenwart, a cura di Han Felten e David Nelting, Frankfurt am Mein, Peter Lang, p. 129-135. 
Di Cuonzo A., 2002, «Storia, menzogna e letteratura in Vincenzo Consolo», «Allegoria», 40-41, p. 164-173.

Di Legami F., 2008, "Vincenzo Consolo tra romanzo e antiromanzo», in $\mathrm{La}$ rappresentazione allo specchio. Testo letterario e testo pittorico, Milano, Booklet, p. 309-320.

Dombroski R. S., 2005, "Consolo and the Fictions of History», in: Risorgimento in Modern Italian Culture, a cura di Norma Bouchard, Madison-Teanecke, Fairleigh Dickinson University Press, p. 217-237.

Fracchiola J., 2008, «Storia e storie nell'opera di Vincenzo Consolo», in: La pasión por la lengua: Vincenzo Consolo, a cura di Irene Romera Pintor, Valencia, Generalitat valenciana y Universitat de Valencia.

Imberty C., 2007, «Vincenzo Consolo, ou le roman entre mémoire et mémoire historique», in: Vincenzo Consolo, étique et écriture, Paris, Presse Sorbonne Nouvelle.

Messina N., 2005, «Per una storia di Il sorriso dell'ignoto marinaio di Vincenzo Consolo», Quaderns d'Italià, 10, Leggere Vincenzo Consolo/Llegir Vincenzo Consolo, a cura di M.Á. Cuevas.

Messina N., 2007, Per un'edizione critico-genetica dell'opera narrativa di Vincenzo Consolo Il Sorriso dell'ignoto marinaio, Madrid, Universidad Complutense de Madrid.

Ochoa Santos M., 1999, «Historia, ficción y narración en la literatura deVincenzo Consolo», Espéculo.

O'Connell D., 2008, «Consolo narratore e scrittore palincestuoso», Quaderns d'Italià, 13, p. 161-184.

O'Connell D., 2008, "AAnd he a face stillforming”: Genesis Gestation and Variation in Vincenzo Consolo's Il sorriso dell'ignoto marinaio», Italian Studies, 1, 2008, p. 119-140.

Recupero A., 2007, «Sicile 1943-2000: entre la pétrification de l'histoire et l'orageux changement de la société», in Vincenzo Consolo, étique et écriture, Paris, Presses Sorbonne Nouvelle.

Segre C., 2005, «Inserti storiografici e storiografia sotto accusa nel capolavoro di Vincenzo Consolo: Il sorriso dell'ignoto marinaio», in: Segre C., Tempo di bilanci. La fine del Novecento, Torino, Einaudi.

Traina G., 2001, Vincenzo Consolo, Fiesole, Cadmo. 